\title{
The controllability for the internally controlled 1-D wave equation via a finite difference method
}

\author{
Juntao Li ${ }^{1,2}$, Yuhan Zheng ${ }^{1}$ and Guojie Zheng ${ }^{1 *}$
}

\section{"Correspondence:}

guojiezheng@yeah.net

${ }^{1}$ College of Mathematics and Information Science, Henan Normal

University, Xinxiang, 453007, P.R. China

Full list of author information is available at the end of the article

\begin{abstract}
In this paper, we study the controllability of the semi-discrete internally controlled 1-D wave equation by using the finite difference method. In the discrete setting of the finite difference method, we derive the observability inequality and get the exact controllability for the semi-discrete internally controlled wave equation in the one-dimensional case. Then we also analyze whether the uniform observability inequality holds for the adjoint system as $h \rightarrow 0$.
\end{abstract}

MSC: 49K20; 35J65

Keywords: wave equation; finite difference; observability inequality

\section{Introduction}

In this paper, we discuss the topic related to the controllability for the space semidiscretizations of the internally controlled one-dimensional wave equation. Let us first introduce certain notations and state the controlled system as studied in this paper. Let $\omega=(a, b)$ be an open and nonempty subset of $(0,1), \chi_{\omega}$ represent the characteristic function of $\omega$. The controlled wave equation is represented as follows:

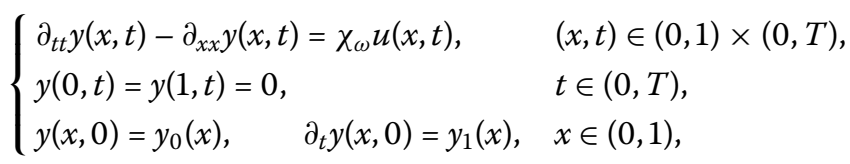

where $T>0$, the initial value $\left(y_{0}, y_{1}\right)$ belongs to $H_{0}^{1}(0,1) \times L^{2}(0,1)$ and $u(\cdot)$ is a control function taken from the space $L^{2}\left(0, T ; L^{2}(0,1)\right)$.

Problem (1.1) is said to be exactly controllable from the initial value $\left(y_{0}, y_{1}\right) \in H_{0}^{1}(0,1) \times$ $L^{2}(0,1)$ in time $T$ if there exists a control function $u(\cdot) \in L^{2}\left(0, T ; L^{2}(0,1)\right)$, such that the solution of $(1.1)$ satisfies $\left(y(T), \partial_{t} y(T)\right)=(0,0)$. The problem of the controllability of wave equations has also been the object of numerous studies. Extensive related references can be found in [1-3] and the rich work cited therein.

In this work, we shall mainly focus on the issue of how the controllability property can be achieved under the numerical approximation schemes. Now, we will introduce the numerical project by using the finite difference method. Given $N \in \mathbb{N}$, we define $h=\frac{1}{N+1}$. We

(c) The Author(s) 2016. This article is distributed under the terms of the Creative Commons Attribution 4.0 International License (http://creativecommons.org/licenses/by/4.0/), which permits unrestricted use, distribution, and reproduction in any medium, provided you give appropriate credit to the original author(s) and the source, provide a link to the Creative Commons license, and indicate if changes were made. 
consider the nodal points

$$
x_{0}=0 ; \quad x_{j}=j h, \quad j=1, \ldots, N ; \quad x_{N+1}=1,
$$

which divides $[0,1]$ into $N+1$ subintervals $I_{j}=\left[x_{j}, x_{j+1}\right], j=0,1, \ldots, N$. We suppose that the nodal points $x_{k+1}, \ldots, x_{k+p} \in \omega$, and $x_{1}, \ldots, x_{k}, x_{k+p+1}, \ldots, x_{N} \in(0,1) \backslash \omega$, for some $k, p \in \mathbb{N}$ with $k+p \leq N$.

Now, we consider the following finite difference semi-discretization of (1.1):

$$
\begin{cases}y_{j}^{\prime \prime}(t)-\frac{1}{h^{2}}\left[y_{j+1}(t)+y_{j-1}(t)-2 y_{j}(t)\right]=\left(\chi_{\omega} u\right)_{j}(t), & t \in(0, T), \\ y_{0}(t)=y_{N+1}(t)=0, & t \in(0, T), \\ y_{j}(0)=y_{j}^{0}, \quad y_{j}^{\prime}(0)=y_{j}^{1}, & j=1, \ldots, N,\end{cases}
$$

where

$$
\left(\chi_{\omega} u\right)_{j}(t)= \begin{cases}u\left(x_{j}, t\right), & \text { if } x_{j} \in \omega, \\ 0, & \text { if } x_{j} \in(0,1) \backslash \omega\end{cases}
$$

The conditions $y_{0}(t)=y_{N+1}(t)=0, t \in(0, T)$, are the Dirichlet boundary conditions in semi-discrete case. Next, we shall rewrite equation (1.2) by vectors. Let

$$
\begin{aligned}
& \vec{y}_{h}(t)=\left(y_{1}(t), \ldots, y_{N}(t)\right)^{T}, \\
& \vec{y}_{h}^{0}=\left(y_{0}\left(x_{1}\right), \ldots, y_{0}\left(x_{N}\right)\right)^{T}, \\
& \vec{y}_{h}^{1}=\left(y_{1}\left(x_{1}\right), \ldots, y_{1}\left(x_{N}\right)\right)^{T},
\end{aligned}
$$

and

$$
\vec{u}_{h}(t)=\left(u_{1}(t), \ldots, u_{N}(t)\right)^{T}
$$

Define the two $N \times N$ matrices

$$
A_{h}=\frac{1}{h^{2}}\left[\begin{array}{ccccc}
2 & -1 & \cdots & 0 & 0 \\
-1 & 2 & \cdots & 0 & 0 \\
\vdots & \vdots & \vdots & \vdots & \vdots \\
0 & 0 & \cdots & 2 & -1 \\
0 & 0 & \cdots & -1 & 2
\end{array}\right]
$$

and

$$
B_{h}=\left[\begin{array}{ccc}
O_{k \times k} & \cdots & O \\
\vdots & I_{p \times p} & \vdots \\
O & \cdots & O_{(N-k-p) \times(N-k-p)}
\end{array}\right],
$$


where $I_{p \times p}$ is a $p \times p$ identity matrix, and $p, k \in \mathbb{N}$, which are mentioned above (1.2). The system (1.2) can be rewritten as follows:

$$
\begin{cases}\vec{y}_{h}^{\prime \prime}(t)+A_{h} \vec{y}_{h}(t)=B_{h} \vec{u}_{h}(t), & t \in(0, T), \\ y_{0}(t)=y_{N+1}(t)=0, & t \in(0, T), \\ \vec{y}_{h}(0)=\vec{y}_{h}^{0}, \quad \vec{y}_{h}^{\prime}(0)=\vec{y}_{h}^{1}, & \end{cases}
$$

where' denotes derivation with respect to time. In fact, system (1.3) is in the form of linear ordinary differential equations for an unknown vector function $\vec{y}(t)_{h}=\left(y_{1}(t), \ldots, y_{N}(t)\right)^{T}$, with the boundary conditions $y_{0}(t)=y_{N+1}(t)=0$, and $\vec{u}_{h}(t)$ plays the role of control function. The adjoint system for system (1.3) can be represented as

$$
\begin{cases}\vec{\phi}_{h}^{\prime \prime}(t)+A_{h} \vec{\phi}_{h}(t)=0, & t \in(0, T), \\ \phi_{0}(t)=\phi_{N+1}(t)=0, & t \in(0, T), \\ \vec{\phi}_{h}(0)=\vec{\phi}_{0}^{h}, \quad \vec{\phi}_{h}^{\prime}(0)=\vec{\phi}_{1}^{h}, & \end{cases}
$$

where the initial date $\vec{\phi}_{0}^{h}=\left(\phi_{1}^{0}, \ldots, \phi_{N}^{0}\right)^{T}$, and $\vec{\phi}_{1}^{h}=\left(\phi_{1}^{1}, \ldots, \phi_{N}^{1}\right)^{T}$. It is easy to check that $\phi_{0}^{0}=\phi_{N+1}^{0}=0$ and $\phi_{0}^{1}=\phi_{N+1}^{1}=0$ are the compatibility conditions. Throughout the paper, we suppose that the compatibility conditions hold for any initial value.

Taking the boundary conditions $\phi_{0}(t)=\phi_{N+1}(t)=0, \forall t \in[0, T]$, for the solution of (1.4) we define the energy of the semi-discrete system (1.4) as

$$
E_{h}(t)=\frac{h}{2} \sum_{i=0}^{N}\left(\left|\phi_{i}^{\prime}(t)\right|^{2}+\left|\frac{\phi_{i+1}(t)-\phi_{i}(t)}{h}\right|^{2}\right), \quad \forall t \in[0, T] .
$$

Since $B_{h}$ is a symmetric matrix, the observability inequality of (1.4) can be formulated as: To find a constant $C(T, h)$ such that (see [4-6])

$$
E_{h}(0) \leq C(T, h) \int_{0}^{T}\left\|B_{h} \vec{\phi}_{h}(t)\right\|_{\mathbb{R}^{N}}^{2} d t
$$

where

$$
E_{h}(0)=\frac{h}{2} \sum_{i=0}^{N}\left(\left|\phi_{i}^{1}\right|^{2}+\left|\frac{\phi_{i+1}^{0}-\phi_{i}^{0}}{h}\right|^{2}\right) .
$$

Remark 1.1 The energy $E_{h}(t)$ is conserved for the solution of (1.4). Namely, for any $h>0$ and solution $\vec{\phi}_{h}(t)$ of (1.4), we have (see [5])

$$
E_{h}(t)=E_{h}(0), \quad \text { for any } t \in[0, T] .
$$

In this paper, we will study whether the inequality (1.5) for adjoint system (1.4) holds. We are also interested in whether the constant $C(T, h)$ is bounded as $h \rightarrow 0$. The main results of the paper are presented as follows.

Theorem 1.1 For any $T>0$, the observability estimate (1.5) for the adjoint system (1.4) holds. 
Remark 1.2 Theorem 1.1 shows that the semi-discrete system (1.2) or (1.3) is controllable for any time $T>0$.

Theorem 1.2 For any $T>0$, we have

$$
\sup _{\text {solution of (1.4) }} \frac{E_{h}(0)}{\int_{0}^{T}\left\|B_{h} \vec{\phi}_{h}(t)\right\|_{\mathbb{R}^{N}}^{2} d t} \rightarrow+\infty, \quad \text { as } h \rightarrow 0 .
$$

To the best of our knowledge, Infante and Zuazua made the first study of this topic in [5]. They studied a controllability result for the semi-discrete 1-D wave equation with boundary control. However, the uniform controllability for the semi-discrete systems in [5] cannot be derived as the discretization parameter $h \rightarrow 0$. The main differences between [5] and our paper are as follows. In [5], the authors focused on a one-dimensional boundary controlled wave equation, and we mainly study the internally controlled 1-D wave equation. In this case, the controller is more complicated than the case with controller on boundary. Regarding other works on this subject, we mention $[4,7,8]$ and [6].

The paper is organized as follows: Section 2 briefly describes some preliminary results on the finite difference scheme. The proofs of Theorem 1.1 and Theorem 1.2 are provided in Section 3.

\section{The finite difference scheme}

In this section, we will discuss the numerical project by the finite difference method. We consider the numerical problem for (1.1) in the state space $\mathbb{R}^{N}$ with the usual Euclidean norm and inner product denoted by $\|\cdot\|_{\mathbb{R}^{N}}$ and $\langle\cdot, \cdot\rangle_{\mathbb{R}^{N}}$, respectively. To this end, we first introduce some properties for the eigenvalues and eigenvectors of the matrix $A_{h}$. The spectrum for $A_{h}$ can be explicitly computed in this case (see [9]). The eigenvalues $\lambda_{i}(h)$ $(i=1, \ldots, N)$ satisfy

$$
\lambda_{i}(h)=\frac{4}{h^{2}} \sin ^{2}\left(\frac{i \pi h}{2}\right)
$$

and the corresponding unit eigenvectors in $\mathbb{R}^{N}$ are

$$
\vec{w}_{i}^{h}=\left(w_{i, 1}^{h}, \ldots, w_{i, N}^{h}\right)^{T}, \quad \text { and } \quad w_{i, j}^{h}=\sqrt{\frac{2}{N+1}} \sin (i \pi j h), \quad j=1, \ldots, N .
$$

Clearly, the family of eigenvectors

$$
\left\{\vec{w}_{1}^{h}, \vec{w}_{2}^{h}, \ldots, \vec{w}_{N}^{h}\right\} \text { forms an orthonormal basis of } \mathbb{R}^{N} \text {. }
$$

Indeed, we can easily get the following properties for these eigenvectors.

\section{Lemma 2.1}

(i) For any eigenvector $w=\left(w_{1}, w_{2}, \ldots, w_{N}\right)^{T}$ with eigenvalue $\lambda(h)$ of matrix $A_{h}$, the following identity holds:

$$
\sum_{j=0}^{N}\left|\frac{w_{j}-w_{j+1}}{h}\right|^{2}=\lambda(h) \sum_{j=1}^{N}\left|w_{j}\right|^{2},
$$

where $w_{0}=w_{N+1}=0$. 
(ii) If $w_{k}=\left(w_{k, 1}, w_{k, 2}, \ldots, w_{k, N}\right)^{T}$ and $w_{l}=\left(w_{l, 1}, w_{l, 2}, \ldots, w_{l, N}\right)^{T}$ are eigenvectors associated to eigenvalue $\lambda_{k}, \lambda_{l}$, and $\lambda_{k} \neq \lambda_{l}$, then we have

$$
\sum_{j=0}^{N}\left(w_{k, j}-w_{k, j+1}\right)\left(w_{l, j}-w_{l, j+1}\right)=0
$$

where $w_{k, 0}=w_{k, N+1}=0$, and $w_{l, 0}=w_{l, N+1}=0$.

This lemma is quoted from [5].

Lemma 2.2 Assume $N$ is large enough so that $\omega$ contains at least two consecutive nodal points. Then

$$
B_{h} \vec{w}_{i}^{h} \neq 0, \quad \text { for all } i=1,2, \ldots, N \text {. }
$$

Proof Since there are more than two consecutive nodal points in $\omega=(a, b)$, we let $l(h)$ denote the first natural number such that $l(h) h \in(a, b)$, and $m(h)$ denote the last natural number such that $m(h) h \in(a, b)$. Then we have

$$
B_{h} \vec{w}_{i}^{h}=\left(0, \ldots, 0, w_{i, l(h)}^{h}, \ldots, w_{i, m(h)}^{h}, 0, \ldots, 0\right)^{T}
$$

Here,

$$
w_{i, l(h)}^{h}=\sqrt{\frac{2}{N+1}} \sin (i \pi l(h) h)=\sqrt{\frac{2}{N+1}} \sin \left(\frac{i l(h) \pi}{N+1}\right),
$$

and

$$
w_{i, l(h)+1}^{h}=\sqrt{\frac{2}{N+1}} \sin (i \pi(l(h)+1) h)=\sqrt{\frac{2}{N+1}} \sin \left(\frac{i(l(h)+1) \pi}{N+1}\right)
$$

could not be zero at the same time. Thus, we complete the proof of the lemma.

Especially, we can get the following property for the eigenvectors for the matrix $A_{h}$, which will play a key role in the proof of the main results.

Proposition 2.1 Assume $N$ is large enough so that $\omega$ contains at least two consecutive nodal points. Then there exists a positive constant $L$ which is independent on $h$ such that

$$
\left\|B_{h} \vec{w}_{i}^{h}\right\|_{\mathbb{R}^{N}}>L
$$

holds for the unit eigenvector $\vec{w}_{i}^{h}(i=1, \ldots, N)$ of the matrix $A_{h}$.

Proof First of all, we claim that there exists a positive number $M$ which is independent on $r$ such that

$$
\int_{a}^{b} \sin ^{2}(r \pi t) d t>M, \quad \text { for } r=1,2, \ldots
$$


To this end, we calculate the following integrations:

$$
\begin{aligned}
\int_{a}^{b} \sin ^{2}(r \pi t) d t & =\frac{b-a}{2}-\frac{\sin (2 r \pi b)-\sin (2 r \pi a)}{4 r \pi} \\
& \rightarrow \frac{b-a}{2}, \quad \text { as } r \rightarrow \infty
\end{aligned}
$$

On the one hand, there exists a positive number $N_{1}$ depending only on $b-a$, such that

$$
\int_{a}^{b} \sin ^{2}(r \pi t) d t>\frac{b-a}{4}, \quad \text { as } r>N_{1}
$$

On the other hand, there exists a positive number $M_{1}$ depending only on $N_{1}$, such that

$$
\int_{a}^{b} \sin ^{2}(r \pi t) d t>M_{1}, \quad \text { as } 0<r \leq N_{1}
$$

Taking $M=\min \left\{\frac{b-a}{4}, M_{1}\right\}$, we see that the claim is correct.

After directly calculating, we obtain

$$
\begin{aligned}
\left\|B_{h} \vec{w}_{i}^{h}\right\|_{\mathbb{R}^{N}}^{2} & =\sum_{j h \in(a, b)} \frac{2}{N+1}[\sin (i \pi j h)]^{2} \\
& =\sum_{j h \in(a, b)} \frac{2}{N+1} \frac{1-\cos (2 i \pi j h)}{2} \\
& =\sum_{j h \in(a, b)} \frac{1}{N+1}-\frac{1}{N+1} \sum_{j h \in(a, b)} \cos (2 i \pi j h) .
\end{aligned}
$$

Let $l(h)$ denote the first natural number such that $l(h) h \in(a, b)$, and $m(h)$ denote the last natural number such that $m(h) h \in(a, b)$. Obviously, we have

$$
l(h)=\left[\frac{a}{h}\right]+1
$$

and

$$
m(h)= \begin{cases}{\left[\frac{b}{h}\right],} & \text { if } \frac{b}{h} \notin \mathbb{N} \\ \frac{b}{h}-1, & \text { if } \frac{b}{h} \in \mathbb{N}\end{cases}
$$

Note that

$$
\frac{1}{N+1} \sum_{j h \in(a, b)} \cos (2 i \pi j h)=\frac{1}{2(N+1)} \frac{\sin ([2 m(h)+1] i \pi h)-\sin ([2 l(h)-1] i \pi h)}{\sin (i \pi h)}
$$

Hence, there exists a natural number $I_{1}$ depending only on $b-a$, such that $\frac{1}{i}<\frac{b-a}{4}$, as $i>I_{1}$. From the theory of classic analysis, we have

$$
\frac{2}{\pi} \leq \frac{\sin x}{x} \leq 1, \quad \text { as } x \in\left(0, \frac{\pi}{2}\right]
$$


i.e.,

$$
\sin x \geq \frac{2}{\pi} x, \quad \text { as } x \in\left(0, \frac{\pi}{2}\right] .
$$

Combining the above inequality with (2.9), we see that

$$
\left|\frac{1}{N+1} \sum_{j h \in(a, b)} \cos (2 i \pi j h)\right| \leq \frac{1}{2(N+1)} \frac{2}{i \pi h}=\frac{1}{i \pi}<\frac{b-a}{4},
$$

If $0<i \leq I_{1}$, then

$$
\sum_{j h \in(a, b)} \frac{2}{N+1}[\sin (i \pi j h)]^{2} \rightarrow 2 \int_{a}^{b} \sin ^{2}(i \pi t) d t
$$

for $i=1,2, \ldots, I_{1}$, as $N \rightarrow \infty$, and

$$
\sum_{j h \in(a, b)} \frac{1}{N+1}=\frac{m(h)-l(h)}{N+1} \rightarrow b-a, \quad \text { as } N \rightarrow \infty .
$$

Thus, there exists a positive number $N_{2}>I_{1}$ depending only on $a, b$ and $b-a$, such that

$$
\sum_{j h \in(a, b)} \frac{1}{N+1}>\frac{b-a}{2}
$$

and

$$
\sum_{j h \in(a, b)} \frac{2}{N+1}[\sin (i \pi j h)]^{2}>M,
$$

for $i=1,2, \ldots, I_{1}$, when $N>N_{2}$.

Case I: $N>N_{2}, N \geq i>I_{1}$. From (2.8), (2.10), and (2.11), we can easily see that

$$
\left\|B_{h} \vec{w}_{i}^{h}\right\|_{\mathbb{R}^{N}}^{2}=\sum_{j h \in(a, b)} \frac{1}{N+1}-\frac{1}{N+1} \sum_{j h \in(a, b)} \cos (2 i \pi j h)>\frac{b-a}{4} .
$$

Case II: $N>N_{2}, 0<i \leq I_{1}$. According to (2.12), we can derive

$$
\left\|B_{h} \vec{w}_{i}^{h}\right\|_{\mathbb{R}^{N}}^{2}=\sum_{j h \in(a, b)} \frac{2}{N+1}[\sin (i \pi j h)]^{2}>M .
$$

Case III: $N \leq N_{2}$. According to Lemma 2.2, there exists a positive constant $L_{1}$, which is independent on $h$, such that

$$
\left\|B_{h} \vec{w}_{i}^{h}\right\|_{\mathbb{R}^{N}}>L_{1}
$$

for any unit eigenvector $\vec{w}_{i}^{h}(i=1, \ldots, N)$.

In summary, taking $L=\min \left\{\frac{b-a}{4}, \frac{M}{2}, L_{1}\right\}$, we can complete the proof of this conclusion. 
Remark 2.1 This theorem gives a fundamental property for the unit eigenvectors $\vec{w}_{i}^{h}$ $(i=1, \ldots, N)$ of the discrete Laplacian operator. It shows that the energy for these unit eigenvectors have an uniform lower boundary, which is positive and not dependent on $h$, in a nonempty and open subset $\omega \subset(0,1)$.

Now, we need to introduce certain notations. Let $X_{0}^{h}$ denote the space $\mathbb{R}^{N}$ equipped with the norm $\|\cdot\|_{0}$

$$
\|u\|_{0}=h \sum_{j=1}^{N}\left|u_{j}\right|^{2}, \quad \text { for any } u=\left(u_{1}, u_{2}, \ldots, u_{N}\right)^{T} \in \mathbb{R}^{N} .
$$

Let $X_{1}^{h}$ denote the space $\mathbb{R}^{N}$ equipped with the norm $\|\cdot\|_{1}$,

$$
\|u\|_{1}=h \sum_{j=0}^{N}\left|\frac{u_{j}-u_{j+1}}{h}\right|^{2}, \quad \text { for any } u=\left(u_{1}, u_{2}, \ldots, u_{N}\right)^{T} \in \mathbb{R}^{N},
$$

where $u_{0}=u_{N+1}=0$. According to the definitions of the discrete norms, the energy can be represented as $E_{h}(t)=\frac{1}{2}\left(\left\|\vec{\phi}_{h}^{\prime}(t)\right\|_{0}+\left\|\vec{\phi}_{h}(t)\right\|_{1}\right)$, where $\vec{\phi}_{h}(t)$ is the solution of equation (1.4).

Lemma 2.3 For any vector $u=\left(u_{1}, u_{2}, \ldots, u_{N}\right)^{T} \in \mathbb{R}^{N}$, we have the following inequality:

$$
\lambda_{1}(h)\|u\|_{0} \leq\|u\|_{1} .
$$

This lemma can easily be deduced from Lemma 2.1.

\section{Remark 2.2}

(i) According to Lemma 2.3, it is easy to find that the spaces $X_{0}^{h}$ and $X_{1}^{h}$ are both Banach spaces. In fact, $X_{0}^{h}$ and $X_{1}^{h}$ can be regarded as the discrete version of the space $L^{2}(0,1)$ and $H_{0}^{1}(0,1)$, respectively. Thus, Lemma 2.3 can be regarded as the discrete version of Poincarés inequality.

(ii) Since $\mathbb{R}^{N} \times \mathbb{R}^{N}$ is a finite dimensional space, thus all norms of this space are equivalent. In particular, there exist positive numbers $C_{1}, C_{2}$, such that

$$
C_{1}\left\|\left(z_{1}, z_{2}\right)\right\|_{X_{0}^{h} \times X_{1}^{h}} \leq\left\|\left(z_{1}, z_{2}\right)\right\|_{\mathbb{R}^{N} \times \mathbb{R}^{N}} \leq C_{2}\left\|\left(z_{1}, z_{2}\right)\right\|_{X_{0}^{h} \times X_{1}^{h}}
$$

hold for any $\left(z_{1}, z_{2}\right) \in \mathbb{R}^{N} \times \mathbb{R}^{N}$.

\section{The proof of Theorem 1.1 and Theorem 1.2}

\subsection{The proof of Theorem 1.1}

Proof First of all, we will prove that there exists a positive constant $C(T, h)$ such that the inequality

$$
\left\|\left(\vec{\phi}_{0}^{h}, \vec{\phi}_{1}^{h}\right)\right\|_{\mathbb{R}^{N} \times \mathbb{R}^{N}}^{2} \leq C(T, h) \int_{0}^{T}\left\|B_{h} \vec{\phi}_{h}(t)\right\|_{\mathbb{R}^{N}}^{2} d t
$$

holds, where $\vec{\phi}_{h}(t)$ is the solution of (1.4) with initial data $\left(\vec{\phi}_{0}^{h}, \vec{\phi}_{1}^{h}\right)$. 
Let $T>0$. We first define a function $F: \mathbb{R}^{N} \times \mathbb{R}^{N} \rightarrow \mathbb{R}$ as

$$
F\left(\vec{\phi}_{0}^{h}, \vec{\phi}_{1}^{h}\right)=\int_{0}^{T}\left\|B_{h} \vec{\phi}_{h}(t)\right\|_{\mathbb{R}^{N}}^{2} d t
$$

where $\vec{\phi}_{h}(t)$ is the solution of (1.4) with initial data $\left(\vec{\phi}_{0}^{h}, \vec{\phi}_{1}^{h}\right)$. Obviously, $F$ is continuous. Now, we will prove that

$$
\min \left\{F\left(\vec{\phi}_{0}^{h}, \vec{\phi}_{1}^{h}\right) ;\left\|\left(\vec{\phi}_{0}^{h}, \vec{\phi}_{1}^{h}\right)\right\|_{\mathbb{R}^{N} \times \mathbb{R}^{N}}=1\right\} \geq L(h, T)
$$

holds for certain positive constant $L(h, T)$ only depending on $h$ and $T$.

Suppose that there exists an unit vector $\left(\vec{\varphi}_{0}^{h}, \vec{\varphi}_{1}^{h}\right)$ in $\mathbb{R}^{N} \times \mathbb{R}^{N}$ such that $F\left(\vec{\varphi}_{0}^{h}, \vec{\varphi}_{1}^{h}\right)=0$. Since $\left\|\left(\vec{\varphi}_{0}^{h}, \vec{\varphi}_{1}^{h}\right)\right\|_{\mathbb{R}^{N} \times \mathbb{R}^{N}}=1, \vec{\varphi}_{0}^{h}$ and $\vec{\varphi}_{1}^{h}$ could not be zero at the same time. Without loss of generality, we assume that $\vec{\varphi}_{0}^{h} \neq 0$. According to (2.3), we have

$$
\vec{\varphi}_{0}^{h}=\sum_{j=1}^{N} \varphi_{0}^{j} \vec{w}_{j}^{h}
$$

and

$$
\vec{\varphi}_{1}^{h}=\sum_{j=1}^{N} \varphi_{1}^{j} \vec{w}_{j}^{h}
$$

where $\sum_{j=1}^{N}\left|\varphi_{0}^{j}\right|^{2}=\left\|\vec{\varphi}_{0}^{h}\right\|_{\mathbb{R}^{N}}^{2} \neq 0$. Solving (1.4), we can deduce that

$$
\vec{\phi}_{h}(t)=\sum_{j=1}^{N} \beta_{j}(t) \vec{w}_{j}^{h}
$$

where $\beta_{j}(t)=\varphi_{0}^{j} \cos \left(\sqrt{\lambda_{j}(h)} t\right)+\frac{\varphi_{1}^{j}}{\sqrt{\lambda_{j}(h)}} \sin \left(\sqrt{\lambda_{j}(h)} t\right)$.

From the definition of the function $F$ and the assumption that $F\left(\vec{\varphi}_{0}^{h}, \vec{\varphi}_{1}^{h}\right)=0$, we have

$$
0=F\left(\vec{\varphi}_{0}^{h}, \vec{\varphi}_{1}^{h}\right)=\int_{0}^{T}\left\|B_{h} \vec{\phi}_{h}(t)\right\|_{\mathbb{R}^{N}}^{2} d t=\int_{0}^{T} \sum_{j=1}^{N}\left|\beta_{j}(t) B_{h} \vec{w}_{j}^{h}\right|^{2} d t
$$

Thus,

$$
\sum_{j=1}^{N} \beta_{j}(t) B_{h} \vec{w}_{j}^{h}=0, \quad \text { for any } t \in[0, T]
$$

It follows from Lemma 2.1 or Proposition 2.1 that $B_{h} \vec{w}_{j}^{h} \neq 0$ for any $j=1,2, \ldots, N$. It is obvious that the rank of subspace spanned by $\left\{B_{h} \vec{w}_{1}^{h}, \ldots, B_{h} \vec{w}_{N}^{h}\right\}$ is less than $N$. Therefore, we can assume that $B_{h} \vec{w}_{1}^{h}, \ldots, B_{h} \vec{w}_{\alpha}^{h}$, with $1 \leq \alpha<N$, are linear independent in $\mathbb{R}^{N}$, and

$$
\operatorname{span}\left\{B_{h} \vec{w}_{1}^{h}, \ldots, B_{h} \vec{w}_{\alpha}^{h}\right\}=\operatorname{span}\left\{B_{h} \vec{w}_{1}^{h}, \ldots, B_{h} \vec{w}_{N}^{h}\right\}
$$


Hence,

$$
B_{h} \vec{w}_{q}^{h}=\sum_{j=1}^{\alpha} b_{q j} B_{h} \vec{w}_{j}^{h}, \quad \text { for any } q=\alpha+1, \ldots, N
$$

For any $q=\alpha+1, \ldots, N$, there exists at least one scalar $b_{q j(q)}(1 \leq j(q) \leq \alpha)$ such that $b_{q j(q)} \neq 0$. This, together with (3.4), indicates that

$$
\begin{aligned}
0 & =\sum_{j=1}^{\alpha} \beta_{j}(t) B_{h} \vec{w}_{j}^{h}+\sum_{q=\alpha+1}^{N} \beta_{q}(t)\left(\sum_{j=1}^{\alpha} b_{q j} B_{h} \vec{w}_{j}^{h}\right) \\
& =\sum_{j=1}^{\alpha}\left(\beta_{j}(t)+\sum_{q=\alpha+1}^{N} \beta_{q}(t) b_{q j}\right) B_{h} \vec{w}_{j}^{h}, \quad \text { for any } t \in[0, T] .
\end{aligned}
$$

According to the linear independence of $\left\{B_{h} \vec{w}_{j}^{h}\right\}_{j=1}^{\alpha}$, we can deduce that

$$
\beta_{j}(t)+\sum_{q=\alpha+1}^{N} \beta_{q}(t) b_{q j}=0, \quad \text { for any } j=1,2, \ldots, \alpha \text {, and for any } t \in[0, T]
$$

Taking $t=0$, we get

$$
\varphi_{0}^{j}+\sum_{q=\alpha+1}^{N} \varphi_{0}^{q} b_{q j}=0, \quad \text { for any } j=1,2, \ldots, \alpha
$$

Differentiating (3.6) twice and taking $t=0$, we have

$$
\lambda_{j}(h) \varphi_{0}^{j}+\sum_{q=\alpha+1}^{N} \lambda_{q}(h) \varphi_{0}^{q} b_{q j}=0, \quad \text { for any } j=1,2, \ldots, \alpha \text {. }
$$

By induction, we obtain

$$
\lambda_{j}^{m}(h) \varphi_{0}^{j}+\sum_{q=\alpha+1}^{N} \lambda_{q}^{m}(h) \varphi_{0}^{q} b_{q j}=0, \quad \text { for any } j=1,2, \ldots, \alpha \text {, and } m \in \mathbb{N}^{+} \text {. }
$$

It follows from (2.1) that $\left\{\lambda_{j}(h)\right\}_{j=1}^{N}$ are different from each other. Thus, we can deduce that

$$
\varphi_{0}^{j}=0, \quad \text { for any } j=1,2, \ldots, \alpha \text {, }
$$

and

$$
\varphi_{0}^{q} b_{q j}=0, \quad \text { for any } q=\alpha+1, \ldots, N \text {, and } j=1,2, \ldots, \alpha
$$

Taking $j=j(q)$, we have

$$
\varphi_{0}^{q}=0, \quad \text { for any } q=\alpha+1, \ldots, N \text {. }
$$


This, together with (3.8), leads to a contradiction to the assumption that $\vec{\varphi}_{0}^{h} \neq 0$. Thus, (3.2) holds. Note that (3.3) implies $F\left(\mu v_{1}, \mu v_{2}\right)=\mu^{2} F\left(\mu v_{1}, \mu v_{2}\right)$ for every $\left(v_{1}, v_{2}\right) \in \mathbb{R}^{N} \times \mathbb{R}^{N}$ and $\mu \in \mathbb{R}$. Thus, it is obvious that inequality (3.2) leads to (3.1).

From (3.1) with (2.13), it is easy to obtain the observability inequality (1.5) of the semidiscrete system (1.4). This completes the proof of this theorem.

\subsection{The proof of Theorem 1.2}

Proof Given the initial data $\vec{\phi}_{0}^{h}=\vec{w}_{N}^{h}$, and $\vec{\phi}_{1}^{h}=0$, the solution of equation (1.4) can be represented as

$$
\vec{\phi}_{h}(t)=\cos \left(\sqrt{\lambda_{j}(h)} t\right) \vec{w}_{N}^{h}
$$

By Lemma 2.1, one shows that

$$
E_{h}(0)=\frac{h}{2} \sum_{i=0}^{N}\left|\frac{w_{N, i+1}-w_{N, i}}{h}\right|^{2}=\frac{h}{2} \lambda_{N}(h)
$$

where $w_{N, 0}=w_{N, N+1}=0$. Then

$$
\begin{aligned}
\int_{0}^{T}\left\|B_{h} \vec{\phi}_{h}(t)\right\|_{\mathbb{R}^{N}}^{2} d t & =\int_{0}^{T}\left|\cos \left(\sqrt{\lambda_{N}(h)} t\right) B_{h} \vec{w}_{N}^{h}\right|^{2} d t \\
& =\left\|B_{h} \vec{w}_{N}^{h}\right\|_{\mathbb{R}^{N}}^{2} \int_{0}^{T}\left|\cos \left(\sqrt{\lambda_{N}(h)} t\right)\right|^{2} d t \leq T .
\end{aligned}
$$

It follows from (3.9) and (3.10) that

$$
\frac{E_{h}(0)}{\int_{0}^{T}\left\|B_{h} \vec{\phi}_{h}(t)\right\|_{\mathbb{R}^{N}}^{2} d t} \geq \frac{\frac{h}{2} \lambda_{N}(h)}{T}=\frac{h \lambda_{N}(h)}{2 T} .
$$

By (2.1), we derive that

$$
\frac{h \lambda_{N}(h)}{2 T}=\frac{2 \sin ^{2}\left(\frac{\pi N h}{2}\right)}{T h} \rightarrow \infty, \quad \text { as } h \rightarrow 0
$$

This completes the proof of this theorem.

\section{Competing interests}

The authors declare that they have no competing interests.

\section{Authors' contributions}

$J L$ provided the questions. YZ and GZ gave the proof for the main result together. All authors read and approved the final manuscript.

\section{Author details}

${ }^{1}$ College of Mathematics and Information Science, Henan Normal University, Xinxiang, 453007, P.R. China. ${ }^{2}$ College of Computer and Information Engineering, Henan University of Economics and Law, Zhengzhou, 450002, P.R. China.

\section{Acknowledgements}

The authors would like to thank the reviewers for their valuable comments and insightful suggestions. This work was partially supported by the National Natural Science Foundation of China (61203293, 61374079), Program for Science and Technology Innovation Talents in Universities of Henan Province (13HASTIT040), Program for Innovative Research Team 
(in Science and Technology) in University of Henan Province (14IRTSTHN023), Henan Higher School Funding Scheme for Young Teachers (2012GGJS-063).

Received: 22 March 2016 Accepted: 15 November 2016 Published online: 24 November 2016

\section{References}

1. Bardos, C, Lebeau, G, Rauch, J: Sharp sufficient conditions for the observation, control and stabilization of waves from the boundary. SIAM J. Control Optim. 30, 1024-1065 (1992)

2. Coron, J-M: Control and Nonlinearity. Mathematical Surveys and Monographs, vol. 136. American Mathematical Society, Providence (2007)

3. Zuazua, E: Controllability and observability of partial differential equations: some results and open problems. In: Handbook of Differential Equations: Evolutionary Equations, vol. 3, pp. 527-621. Elsevier Science, Amsterdam (2006)

4. Castro, C, Micu, S: Boundary controllability of a linear semi-discrete 1-D wave equation derived from a mixed finite element method. Numer. Math. 102, 413-462 (2006)

5. Infante, JA, Zuazua, E: Boundary observability for the space semi-discretization of the 1-D wave equation. ESAIM: Math. Model. Numer. Anal. 33, 407-438 (1999)

6. Zuazua, E: Propagation, observation, control and numerical approximation of waves by finite difference method. SIAM Rev. 47, 197-243 (2005)

7. Castro, C, Micu, S: Numerical approximation of the boundary control for the wave equation with mixed finite elements in a square. IMA J. Numer. Anal. 28, 186-214 (2008)

8. Micu, S, Zuazua, E: An introduction to the controllability of partial differential equations. In: Sari, T (ed.) Collection Travaux en Cours Hermannin Quelques Questions de Théorie du Contrôle, pp. 67-150 (2005)

9. Isaacson, E, Keller, HB: Analysis of Numerical Methods. Wiley, New York (1966)

\section{Submit your manuscript to a SpringerOpen ${ }^{\ominus}$ journal and benefit from:}

- Convenient online submission

Rigorous peer review

- Immediate publication on acceptance

- Open access: articles freely available online

- High visibility within the field

- Retaining the copyright to your article 